\title{
Lesion Segmentation in Breast Sonography
}

\author{
Joan Massich ${ }^{1, \star}$, Fabrice Meriaudeau ${ }^{2}$, Elsa Pérez ${ }^{3}$, Robert Martí ${ }^{1}$, \\ Arnau Oliver ${ }^{1}$, and Joan Martí ${ }^{1}$ \\ 1 Computer Vision and Robotics Group, University of Girona, Spain \\ jmassich@atc.udg.edu \\ 2 University of Burgundy, Le2i Laboratory CNRS UMR 5158, Esplanade Erasme, \\ 21000, Dijon, France \\ ${ }^{3}$ Dept of Radiology, Hospital Josep Trueta of Girona, Spain
}

\begin{abstract}
Sonography is gaining popularity as an adjunct screening technique for assessing abnormalities in the breast. This is particularly true in cases where the subject has dense breast tissue, wherein widespread techniques like Digital Mammography (DM) fail to produce reliable outcomes. This article proposes a novel and fully automatic methodology for breast lesion segmentation in B-mode Ultra-Sound (US) images by utilizing region, boundary and shape information to cope up with the inherent artifacts present in US images. The proposed approach has been evaluated using a set of sonographic images with accompanying expert-provided ground truth.
\end{abstract}

Keywords: ultrasound, breast cancer, segmentation, segmentation evaluation.

\section{Introduction}

Breast cancer is one of the leading causes of death for women in developed countries and is most effectively treated when detected at an early stage [5].

Considering this, DM is, and remains the major screening tool for breast cancer [3. However, in the recent past, studies [6] have shown that US images of the breast can help supplement mammography by detecting breast cancers that may not be visible in a traditional mammogram. This is particularly true in the cases where the subjects have dense glandular breast tissue, which tends to shield the presence of a tumor in a mammogram. In addition, US images are noninvasive with no side effects, rendering sonography as an attractive adjunct to digital mammography and heading a re-emergence of interest in understanding how to do image segmentation applied to ultrasound data [4].

This reserach proposes a novel and fully automatic technique to segment breast lesions for conventional B-mode US images, by utilizing region, boundary and shape information to cope with the inherent artifacts present in these images.

* We gratefully acknowledge the help of Dr. Gururajan and Dr. Sari-Sarraf from Texas Tech University. This research was partially supported by the Spanish Government MEC grant nb. TIN2007-60553 and the University of Girona BR grant nb. 09/22. 


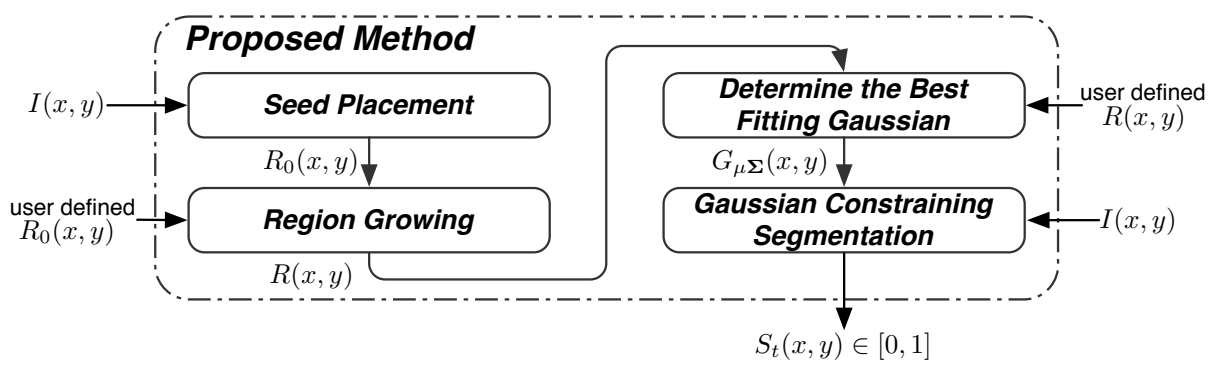

Fig. 1. Block diagram of the proposed methodology. When user interaction is used (only for semi-automatic segmentation), it overwrites the previous input.

The performance evaluation of the presented segmentation approach is conducted using a set of sonographic breast images that have accompanying ground truth provided by multiple experts. In this regard, this paper also presents a modified version of the Simultaneous Truth and Performance Level Estimation (STAPLE) 8, algorithm that is employed to extract the gold-standard for an image that has been delineated by multiple experts.

\section{Proposed Approach}

In this paper, a new segmentation procedure for breast lesions US images is proposed, allowing different levels of user-interaction, and ultimately leading automation of the entire approach. The block diagram of the proposed approach is shown in Fig.11. The primary step involved in this process is the Gaussian Constraining Segmentation (GCS), which is a boundary-based technique whose key input is a multivariate Gaussian function that describes the shape, position and orientation of the lesion, derived from the one proposed by Horsch et al. 1] This Gaussian function is determined by fitting an ellipse to the $R(x, y)$ blob. This $R(x, y)$ preliminar segmentation of the lesion can be an interactive segmentation input or it can be determined by a fuzzy region growing from a seed region $R_{0}(x, y)$. Such region initialization $R_{0}(x, y)$, can also be a given input or it can be automatically determined by the seed placement procedure (a simplification to the seed placement proposed by Madabhushi and Metaxas 2]) leading to a fully automatic segmentation procedure.

The GCS step (see Fig.22), outputs a binary mask in accordance with Eq. (11) and this mask corresponds to the final segmentation result. The GCS consists of two steps: (a) finding $\Psi(x, y)$ which is an intensity dependent function $(f(I(x, y)))$ constrained by a Gaussian $\left(G_{\boldsymbol{\mu} \boldsymbol{\Sigma}}(x, y)\right)$ that best represents the lesion, and (b) selecting the proper threshold for $\Psi(x, y)$ to segment the lesion.

$$
S_{t}(x, y)=\operatorname{threshold}(\Psi(x, y), t) \quad \text { where } \quad \Psi(x, y)=f(I(x, y)) \cdot G_{\mu \Sigma}(x, y)
$$




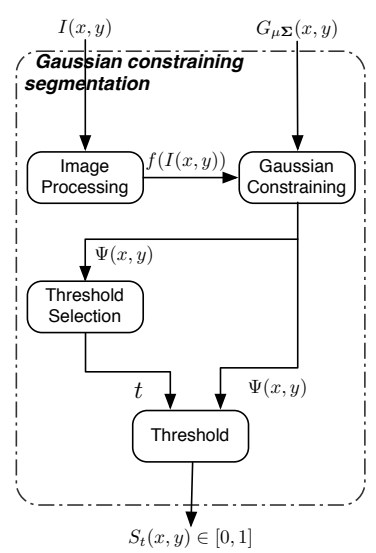

(a)

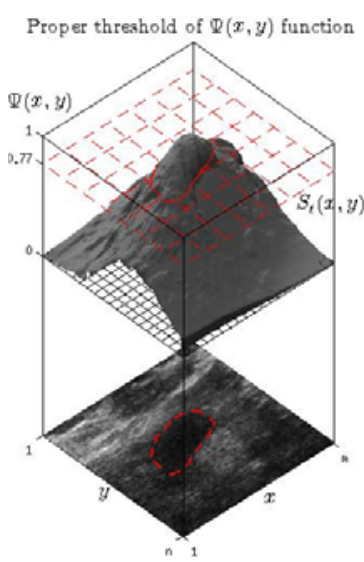

(b)

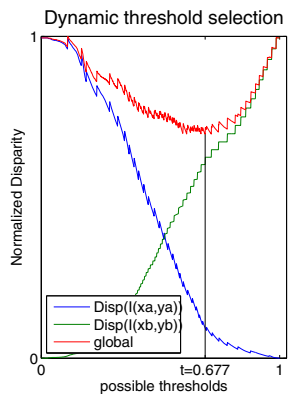

(c)

Fig. 2. Gaussian Constraining Segmentation (GCS) outline: (a) block diagram (b) graphical representation for the GCS The figure shows a 3D representation of the lesion shaped potential funcion $\Psi(x, y)$, and its projection on the sonogram obtained by thresholding $\Psi(x, y)$. (c) threshold selection upon the minimization of the global disparity measure.

For a given dataset, threshold $t$ can either be manually tuned up for the whole set, or dynamically determined for each image based on its characteristics. When a dynamic thresholding is used, the threshold $t$ is selected from a set $\left\{t_{1}, . ., t_{n}\right\}$ as the best candidate accordingly to equation 3 .

$$
\begin{array}{r}
t=\min _{t}\left(\operatorname{Disparity}\left(I\left(x_{u}, y_{u}\right)\right)+\operatorname{Disparity}\left(I\left(x_{d}, y_{d}\right)\right)\right) \\
\text { where } \quad\left(x_{u}, y_{u}\right) \in\{(X, Y) \mid \Psi(x, y) \geq t\} \\
\left(x_{d}, y_{d}\right) \in\{(X, Y) \mid \Psi(x, y)<t\}
\end{array}
$$

Figure2(c) illustrates how the disparity measure varies along the different thresholds. The optimal threshold for each image given the lesion shaped potential function $\Psi(x, y)$ corresponds to the minimum global disparity.

The main advantage of the proposed method over that presented by Horsch et al. 1] is: (a) fully automatic capability without any information of the lesion, (b) better lesion description and delineation. Lastly the advantage over the reserarch done by Madabhushi and Metaxas [2, is the incorporation of a faster lesion refinement step after the region growing.

\section{Experiment Description}

In order to evaluate the proposed approach, a set of 25 sonograms were acquired in the Hospital Dr. Josep Trueta of Girona. Each image has seven ground 
truth delineations provided by different radiology experts. In the first step, the STAPLE algorithm [8] is used to obtain the underlying ground truth from the multiple expert delineations. STAPLE states that the ground truth and performance levels of the experts can be estimated by formulating the scenario as a missing-data problem, which can be subsequently solved using an Expectation Maximization (EM) algorithm. The EM algorithm, after convergence, provides the Hidden Ground Truth (HGT) estimation that has been inferred from the segmentations provided by the experts.

The proposed segmentation approach is evaluated as follows. For a given image, the segmentations produced by the evaluated methods and STAPLE on the $i$ th pixel can be denoted as $S_{i}$ and $G_{i}$ respectively. Within this framework, we propose a performance coefficient based on the True-Positive Ratio (TPR) or Jaccard coefficient weighting its numerator by the HGT Such coefficient is calculated accordingly to Eq. (3), where $p_{i}$ is the HGT probability of each pixel.

$$
\text { coeff }=\frac{\sum\left(S_{i} \cap G_{i}\right) \cdot p_{i}}{\sum\left(S_{i} \cup G_{i}\right)}
$$

For evaluation purposes, the results obtained by applying the proposed approach (on its three user interaction levels: no user input, given a seed region $R_{0}(x, y)$, and, given a blob $R(x, y)$ ) have been compared to the results obtained by applying other techniques: Horsch et al. 1] (on its partially automatic version which needs the lesion center and the four corners), Madabhushi and Metaxas [2. The proposed approach has been tested both with dynamic and manually tuned-up threshold selection.

\section{Results}

The results and dicussion will focus on the performance of the proposed segmentation technique versus that presented in [1 2] over the entire dataset.

Figure 3 shows the segmentation results on four sonograms from the dataset. In the figure, the white dashed line indicates the delineation of the lesion after the segmentation, while the different $\Psi(x, y)$ isolines are represented by means of colored curves.

Previous to the segmentation procedure, a rank value filtering method is applied by means of a $3 \times 3$ median filter, in order to reduce the impact of speckle noise.

To quantitatively assess the segmentation approach, the metric proposed in equation 3 is used to compare the agreement between the gold-standard and the obtained segmentation. This is done for all the user interaction levels available in our proposal and for the whole dataset. The distribution of the dataset segmentation performance or agreement reward, is illustrated as a box-plot in figure 4 . The first six entries represent our method results, differentiating manual tune up thresholding from dynamic selection thresholding for each level of user interaction level. The last two entries, plot the performance of the bibliographic methods 12 . 


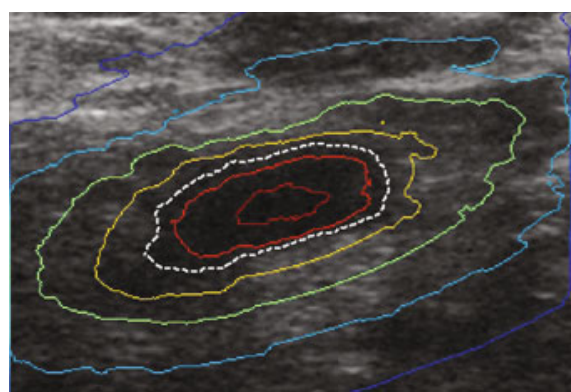

(a)

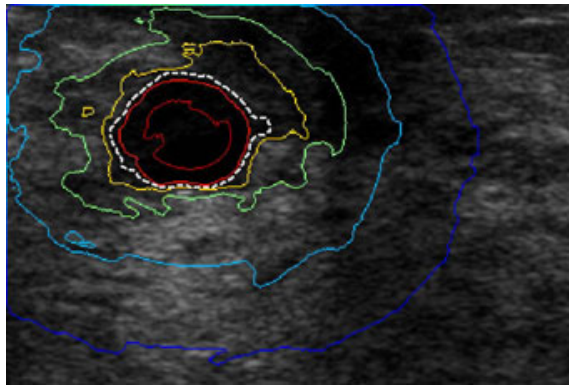

(c)

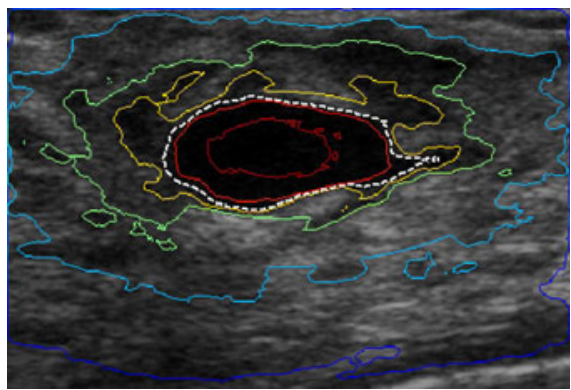

(b)

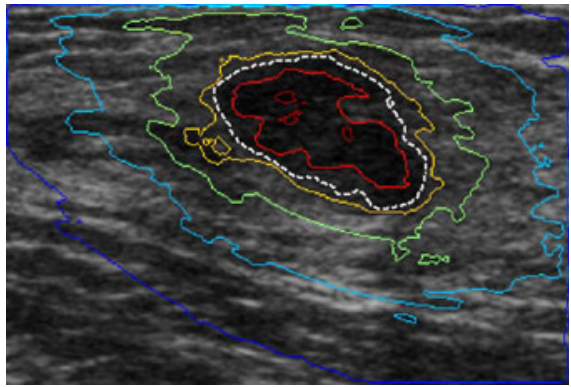

(d)

Fig. 3. Lesion segmentation results for 3 different images from the used dataset. Different level slices of the $\Psi(x, y)$ function are represented by different colour. The white dashed line indicates the lesion segmentation.

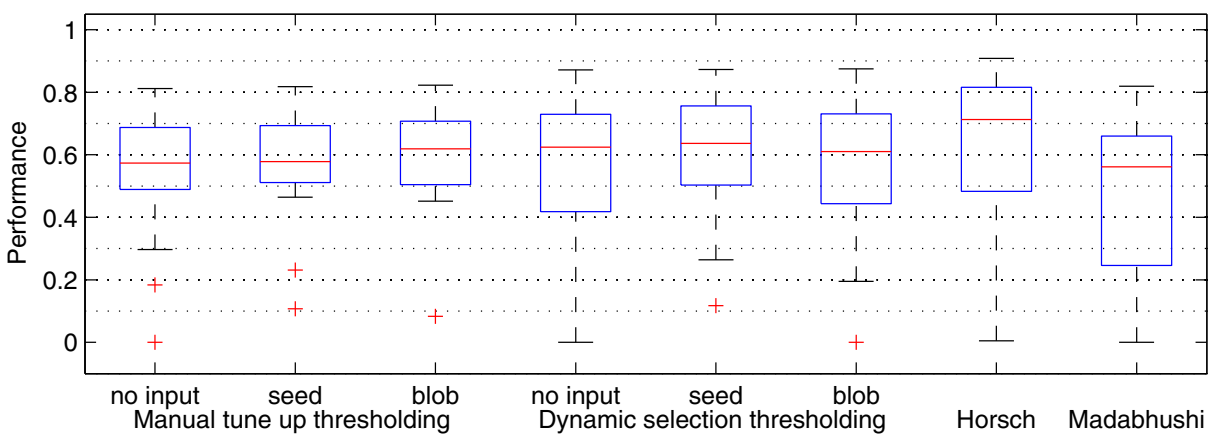

Fig. 4. Box-plot representation of the tested methods performance for the current dataset 


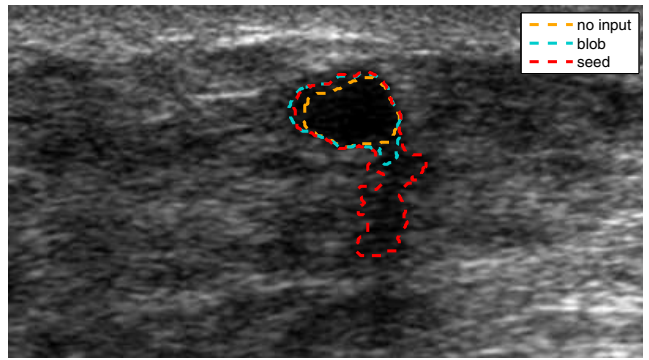

(a)

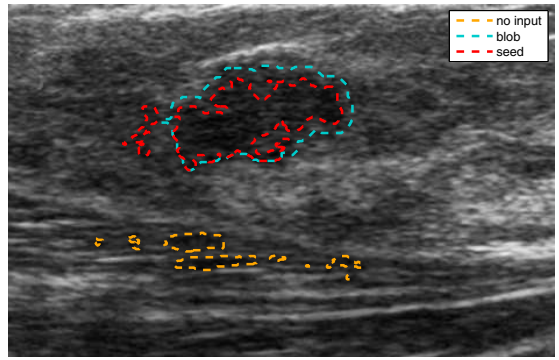

(b)

Fig. 5. Segmentation difficulties: (a) shadowing problem, (b) non-lesion structures segmentation

The results show that the segmentations obtained by the proposed method fit the gold-standard up to $85 \%$ in some cases, while the average values are around $60 \%$. Figure 4 shows that there is less variability when a manually tuned-up threshold is selected. However, the mean values for the segmentation results are higher when thresholding is dynamic.

While comparing the proposed method with the methods from the bibliography [12], our method performs slightly better than the one proposed by Madabhushi and Metaxas 22. On the other hand, although the method proposed by Horsch et al. [1] shows better performance when considering the mean value, our method behaves better in terms of variability.

The quantitative comparison has been performed using the metric proposed in equation 3. Notice that the obtained results are lower than the expected ones when using a TPR metric, because the metric used penalizes or rewards depending on which part of the segmentation $S$ overlaps the gold-standard $G$. The metric used only behaves as TPR when all the elements of the overlapping area have probability 1 of being lesion (see eq.(4).

$$
\frac{\sum\left(S_{i} \cap G_{i}\right) \cdot p_{i}}{\sum\left(S_{i} \cup G_{i}\right)}=\mathrm{TPR} \quad \text { if and only if } \quad p_{i}=1 \forall i
$$

\section{Discussion}

The shadowing problem, as shadow being part of the lesion, is not particularly addressed or treated here. However, as long as the region growing output does not contain the shadow, the proposed method does not consider that shadow as a part of the lesion by means of the multivariate attenuation of the gaussian. Figure 5(a) illustrates how the shadow gets included as a part of the lesion for a seed guided segmentation.

This misdelineation is explained by the region growing initialization $R_{0}(x, y)$ (supplied by the user) since it has lead to grown up region $R(x, y)$ containing the shadow. Subsequently this region $R(x, y)$ has lead to a wrongly fitted gaussian $\left(G_{\mu \Sigma}(x, y)\right)$ that misdescribes the lesion. 
In the same way, the proposed method does not explicitly address the problem of segmenting other non-lesion structures present in the sonogram (like glandular or fatty tissue) as a lesion. Our does not address a situation where the seed region is misplaced on a structure that has some similarities to the lesions. For the present experiment, this only has happened in one image of the dataset. Figure[5(b) shows the sonogram where the automatic seed placement misplace the initial region for the region growing. Hence, the obtained segmentation by using the fully automatic version of the method results in a muscular region being extracted as a lesion. However, in most of the cases the seed region is correctly placed, so the method does not segment structures other than the lesions.

This research has presented and tested a breast lesion segmentation framework or methodology applied to ultrasound images. Although the results are satisfactory, further research of all the variable elements of the proposed method should be done to improve the results. For the testing, the same experiment can be done with a larger dataset to achieve more reliable conclusions.

\section{References}

1. Horsch, K., Giger, M.L., Venta, L.A., Vyborny, C.J.: Automatic segmentation of breast lesions on ultrasound. Medical Physics 28, 1652-1659 (2001)

2. Madabhushi, A., Metaxas, D.: Automatic boundary extraction of ultrasonic breast lesions. In: Proceedings of 2000 IEEE International Symposium on Biomedical Imaging, pp. 601-604 (2002)

3. Moore, S.K.: Better breast cancer detection. IEEE Spectrum 38(5), 50-54 (2001)

4. Noble, J.A., Boukerroui, D.: Ultrasound image segmentation: A survey. IEEE Transactions on medical imaging 25(8), 987-1010 (2006)

5. Sivaramakrishna, R., Powell, K.A., Lieber, M.L., Chilcote, W.A., Shekhar, R.: Texture analysis of lesions in breast ultrasound images. Computerized medical imaging and graphics 26(5), 303-307 (2002)

6. Stavros, A.T., Rapp, C.L., Parker, S.H.: Breast ultrasound. Lippincott Williams \& Wilkins (2004)

7. Taylor, K.J.W., Merritt, C., Piccoli, C., Schmidt, R., Rouse, G., Fornage, B., Rubin, E., Georgian-Smith, D., Winsberg, F., Goldberg, B., et al.: Ultrasound as a complement to mammography and breast examination to characterize breast masses. Ultrasound in Medicine \& Biology 28(1), 19-26 (2002)

8. Warfield, S.K., Zou, K.H., Wells, W.M.: Simultaneous truth and performance level estimation (STAPLE): an algorithm for the validation of image segmentation. IEEE Transactions on Medical Imaging 23(7), 903-921 (2004) 